\title{
A jump that would prove Newton wrong
}

On 21 March - the spring equinox - something very strange may have happened. In two particular places on Earth, objects might have started to move without any force acting on them.

Such motion would violate Newton's second law, a fundamental principle of mechanics, which states that objects accelerate by an amount proportional to the force acting on them. But it needn't involve anything paranormal. According to Alexander Ignatiev of the University of Melbourne in Australia, this weird phenomenon would be proof of a new kind of mechanics called modified newtonian dynamics (MOND).

First suggested in 1983 by Israeli physicist Moti Milgrom, MOND aims to provide an alternative to dark matter, the invisible and so far unidentified stuff invoked by physicists to explain why rotating galaxies don't fall apart.

MOND is very speculative, but interest in it has been boosted in recent years by the observation that the Pioneer 10 and 11 spacecraft, launched in the early 1970s to study the planets, seem to be veering from their expected paths as they leave the Solar System. No one knows whether the anomaly is real or just the result of faulty observations of the spacecraft's motion.

Finding evidence for MOND would be hugely important: "The foundations of physics would have to be revised," says Ignatiev. But measuring the predicted deviations from Newton's theory is extremely difficult. MOND diverges from newtonian dynamics only at very small accelerations, around a hundred trillionths of a metre per second per second. At this rate, an object that began to move when Newton published his second law in 1687 would now be travelling at one metre per second.

To check out the theory, Ignatiev says, you need a test object moving with an acceleration

\section{"The foundations of physics would have to be revised."}

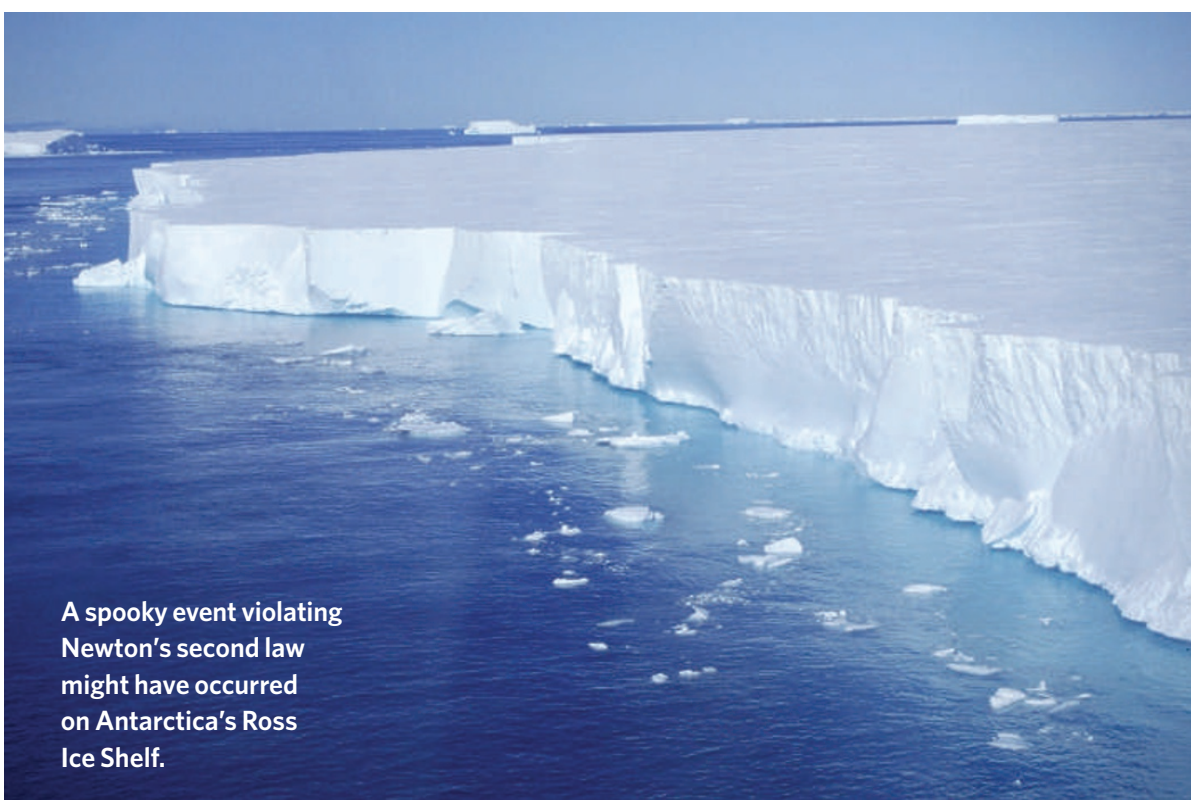

whereas the longitude varies each year. This spring they will be at $178^{\circ} \mathrm{E}$, high above Siberia in the Arctic Sea and on the Ross Ice Shelf of Antarctica. The autumn equinox of 2008 will be a little more amenable to experimenters, when the northern point is in Greenland.

Ignatiev cautions, however, that these are approximate locations, and ignore the subtle effects of the Moon and planets. Before you make the journey, he says, you had better do the full calculation, as the effect acts over an area just a few centimetres across.

So what might you hope to see? As external acceleration effects cancel out, says Ignatiev, the object's acceleration would fall below the MOND threshold - and if the theory is correct, it will spontaneously jump over a small distance.

The predicted jump is tiny: about $0.2 \times 10^{-16}$ metres, or one-

fiftieth of the diameter of a proton. But Ignatiev reckons it could be measured by interferometry, which detects minute differences in the path lengths of light beams. The technique is being developed in huge instruments for detecting gravitational waves, which are predicted to alter the dimensions of space very slightly as they pass. Gravitational-wave detectors typically involve light corridors several kilometres long. But there are now other types of detector that are more portable, and which it might be possible to carry to Antarctica or Greenland. The MiniGRAIL detector being developed at Leiden University in the Netherlands, for example, is a metal ball 68 centimetres in diameter.

Others in the field are sceptical about Ignatiev's chances. Orfeu Bertolami, a physicist at the Instituto Superior Técnico in Lisbon, says that although the proposal is "a brave attempt" to suggest how MOND could be tested, he is not convinced it would work. He points out that Ignatiev used newtonian mechanics to calculate the points of zero acceleration, and that these rules wouldn't necessarily apply in the MOND world he wants to test.

Even if the theory works, Bertolami doubts it would be possible to observe exact cancellation of acceleration. A medium-sized iceberg passing 10 kilometres away in the Antarctic or Arctic Ocean, he says, would induce a gravitational acceleration comparable to the MOND threshold.

And the MOND hypothesis itself is looking decidedly shaky, because there is now rather good evidence that dark matter, which MOND attempts to eliminate, does exist. Last year, a galaxy cluster was found to have an asymmetrical distribution of visible matter that could only easily be explained if it was balanced by dark matter (see Nature doi:10.1038/ news060821-6; 2006). After this, says Bertolami, "any attempt to force MOND on us is difficult to swallow".

But Ignatiev argues that the debate is far from settled. "Experimental searches for dark matter are conducted in many laboratories across the world," he says. "MOND should be given the same chance."

Philip Ball
2007). The points of cancellation are always at latitudes $79^{\circ} 50^{\prime}$ above and below the Equator,

\section{ON MARS}

caverns on the red planet.

www.nature.com/news for MOND effects to be apparent. Even if an erally accelerating with respect to the Galaxy Sun, and the Sun's motion in space.

a particular moment each year, at two points on half a millisecond This, he with either the spring or autumn equinox

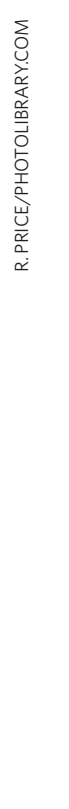

